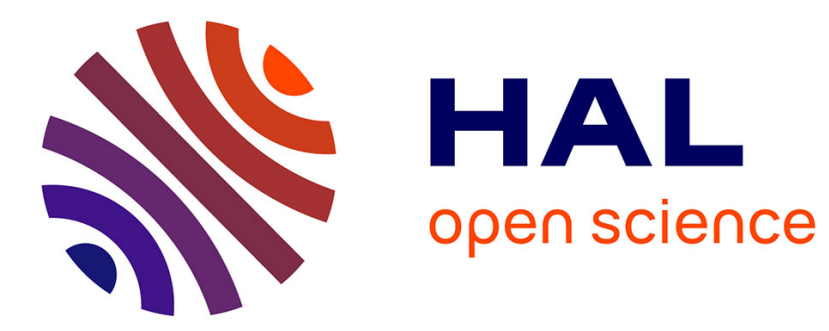

\title{
Preparation and cytotoxicity of lipid nanocarriers containing a hydrophobic flavanone
}

\author{
Daniele Simão, Thalita Honorato, Graciely Gobo, Henrique Piva, Patrícia \\ Goto, Larissa Araújo Rolim, Cédric-Olivier Turrin, Muriel Blanzat, Antonio \\ C. Tedesco, Marigilson Siqueira-Moura
}

\section{To cite this version:}

Daniele Simão, Thalita Honorato, Graciely Gobo, Henrique Piva, Patrícia Goto, et al.. Preparation and cytotoxicity of lipid nanocarriers containing a hydrophobic flavanone. Colloids and Surfaces A: Physicochemical and Engineering Aspects, 2020, 601, pp.124982. 10.1016/j.colsurfa.2020.124982 . hal-02920400

\section{HAL Id: hal-02920400 \\ https://hal.science/hal-02920400}

Submitted on 9 Dec 2020

HAL is a multi-disciplinary open access archive for the deposit and dissemination of scientific research documents, whether they are published or not. The documents may come from teaching and research institutions in France or abroad, or from public or private research centers.
L'archive ouverte pluridisciplinaire HAL, est destinée au dépôt et à la diffusion de documents scientifiques de niveau recherche, publiés ou non, émanant des établissements d'enseignement et de recherche français ou étrangers, des laboratoires publics ou privés. 
Preparation and cytotoxicity of lipid nanocarriers containing a hydrophobic flavanone

Daniele O. Simão ${ }^{\mathrm{a}}$, Thalita D. Honorato ${ }^{\mathrm{b}}$, Graciely G. Gobo ${ }^{\mathrm{c}}$, Henrique L. Piva ${ }^{\mathrm{c}}$, Patrícia

L. Goto ${ }^{d}$, Larissa A. Rolim ${ }^{\mathrm{a}, \mathrm{b}}$, Cédric-Olivier Turrin ${ }^{\mathrm{d}}$, Muriel Blanzat ${ }^{\mathrm{d}}$, Antonio C. $\operatorname{Tedesco}^{\mathrm{c}}$, Marigilson P. Siqueira-Moura ${ }^{\mathrm{a}, \mathrm{b}, *}$

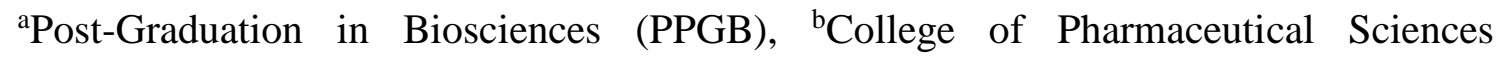
(CFARM), Federal University of the Sao Francisco Valley (UNIVASF), Petrolina, Pernambuco 56304-205, Brazil

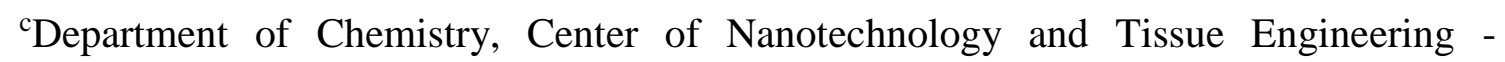
Photobiology and Photomedicine Research Group, Faculty of Philosophy, Sciences and Letters of Ribeirao Preto, University of Sao Paulo (USP), Ribeirao Preto, São Paulo 14040-901, Brazil

dIMRCP Laboratory, UMR 5623 CNRS, Paul Sabatier University, 31062 Toulouse, France

*Corresponding author:

Prof. Marigilson Pontes de Siqueira Moura, Ph.D.

College of Pharmaceutical Sciences - UNIVASF

Prédio dos Colegiados, Av. José de Sá Maniçoba, S/N, 56304-917, Centro, Petrolina-PE, Brazil

Phone: +55 87 21016862, E-mail address: marigilson.moura@univasf.edu.br 


\begin{abstract}
Hesperetin is a flavanone with recognized biological activities. However, such activities are limited due to its restricted aqueous solubility and stability. In this regard, the main aim of this study was to develop and characterize lipid nanocarriers containing hesperetin. Nanostructured lipid carriers (NLC) were prepared using phase inversion temperature method and characterized by size, polydispersity index (PdI), zeta potential, physical stability, TEM analysis, encapsulation efficiency, in vitro release, and in vitro cytotoxic effect in cell line. Lipid nanocarriers presented diameter below $80 \mathrm{~nm}$, narrow $\operatorname{PdI}(<0.2)$, and negative zeta potential $(-20 \mathrm{mV})$. Accelerated stability studies of NLC demonstrated good physical stability for a period of 12 months. According to TEM, NLC were almost spherical with particle size $<100 \mathrm{~nm}$ and homogeneous size distribution. DSC curves showed that formulations presented lipid core with a higher degree of crystalline disorder. Lipid nanocarriers were able to entrap hesperitin with efficiency $72.7 \%( \pm 0.92)$. In vitro release studies confirmed that NLC could modulate hesperetin release during $72 \mathrm{~h}$. In vitro cytotoxicity assay of hesperitin-loaded NLC on T98G glioblastoma grade IV cells presented significant cytotoxic effect. Therefore, NLC were able to encapsulate successfully hesperetin and demonstrated excellent in vitro cytotoxicity on glioblastoma cells.
\end{abstract}

Keywords: Hesperitin, Nanostructured lipid carriers, PIT method, Characterization, Glioblastoma, Cytotoxicity. 


\subsection{Introduction}

Malignant gliomas are the most common primary brain tumors responsible for $80 \%$ of diagnosed cases and glioblastoma multiforme, which is a subcategory of malignant gliomas, is the most aggressive and lethal brain cancer. The poor prognosis of glioblastoma is mainly related to cell drug-resistance and low bioavailability of drugs for brain $[1,2]$. In this regard, researches in order to discover new drugs against glioblastoma become an urgent need in the treatment of brain cancer.

Polyphenolic compounds are ubiquitous in plants and classified as flavonoids, phenolic acids, coumarinea, lignans, tannins, and stilbenes [3,4]. Hesperitin (3',5,7-trihydroxy-4'methoxyflavanone) is a bioflavonoid belonging to the chemical sub-class flavanone, which is widely found in citrus fruits (grapefruit, orange, and lemon). Hesperitin and its glycoside (hesperidin) have demonstrated several biological activities confirmed by in vivo and in vitro studies [4]. Among therapeutic effects attributed to hesperetin, neuroprotection in experimental models of Alzheimer's disease, reduction of blood plasma glucose, lipid levels and antioxidant activity, anti-inflammatory, and antineoplastic activity are the most common effects observed [5-8]. Recently, the potential of antiproliferative and antitumor activities for hesperitin and hesperidin on in vitro and in vivo cancer models has been related to mechanisms of cell cycle arrest and apoptosis [9]. However, in spite of several pharmacological activities of these flavanones, hesperidin and hesperetin have low solubility in water $(<0.01 \%)$ decreasing biological absorption due to limited dissolution rate and consequently low bioavailability [3,10-12]. Another factor that influences their absorption is the presence of microorganisms and enzymes in the small intestine and first-pass liver metabolism, which are able to degrade the active substances into inactive molecules. In addition, environmental factors such as $\mathrm{pH}$ changes, light, and temperature may affect their physical-chemical stability $[13,14]$. 
A possible solution to overcome these problems, increasing flavonoid stability and bioavailability, is the entrapment of such compounds in nanostructures well designed lipid nanoparticles. These colloidal particles are formed by solid lipid core at body temperature stabilized by surfactant(s) and dispersed in aqueous phase [3,15-18]. Lipid nanocarrier systems are composed of submicron particles designed from biocompatible and biodegradable lipids. Solid lipid nanoparticles (SLN) represent the first-generation of lipid particles and are characterized by a core formed by solid lipid(s) at room and body temperature. On the other hand, nanostructured lipid carriers (NLC), which are considered the second-generation of lipid nanocarriers, are formed by unstructured solid core containing a mixture of liquid and solid lipids at room and body temperature $[15,16,18,19]$. The main advantage of NLC is its higher drug loading capacity as compared to SLN, i.e. a lipid core formed by blend of solid and liquid lipids results in low expulsion of encapsulated drug due to formation of more irregular crystals of solid lipid(s). Furthermore, NLC are also able to protect the entrapped active compounds against chemical degradation in physiological $\mathrm{pH}$ [17-19]. Several administration routes have been used to NLC, for instance, oral, topical, ocular, and directly to the brain. SLN and NLC have shown to be effective as brain drug delivery systems promoting the penetration of active substances into the nervous tissue [17,19].

Lipid nanoparticles have been usually produced by high-energy or low-energy emulsification methods [20-24]. High-energy approaches are able to generate intense disruptive forces using mechanical devices such as high-shear stirrers, high-pressure homogenizers, and ultrasound generators. However, high-energy methods require very high energy level to produce fine particles and most of the mechanical energy generated $(99.9 \%)$ is dissipated as heat $[25,26]$. On the other hand, low-energy emulsification approaches are more energy efficient producing small particles by modification of the 
internal chemical energy from system due to temperature or composition change $[20,26,27]$. Low-energy emulsification methods are classified as spontaneous emulsification (SE), phase inversion composition (PIC), emulsion inversion point (EIP), and phase inversion temperature (PIT) [22,27]. The main advantages to obtain lipid nanoparticles using PIT method are very easy production process through simple stirring and heating as well as this method does not require the use of expensive equipment $[20,22,27]$.

Different drug delivery systems have been developed to encapsulate hesperitin or hesperidin, such as polymeric nanoparticles [12,14] and lipid nanoparticles [28-30]. Even if in these studies using high-energy emulsification methods have been commonly reported the successful application of nanocarriers containing flavanones, low-energy methods are more rarely applied to produce lipid nanoparticles although low-energy approaches are considered simple and reproducible methods without using device and/or organic solvent. Thereby, we described hesperitin encapsulation into lipid nanoparticles, which was carried out through thermal low-energy method, and the in vitro potential of this hesperitin-loaded nanocarrier in the inactivation of neoplastic cells from brain cancer. Therefore, the main aim of this study was to develop a promising formulation based on NLC containing hesperitin using PIT method as a low-energy emulsification approach and to assess in vitro cytotoxicity of this encapsulated flavanone on T98G glioblastoma cells. 


\subsection{Materials and methods}

\subsection{Materials}

Hesperitin ( $\geq 95 \%$, W431300), sorbitan monooleate (SMO), medium chain triglycerides (MCT), and dialysis tubing cellulose membrane flat (MW cut-off 14,000) were purchased from Sigma-Aldrich Co. (St. Louis, MO). POE-40 hydrogenated castor oil (HCO-40) was kindly supplied from Croda Brasil Ltda (Campinas-SP, Brazil) and glyceryl behenate (GB) solid lipid was a gift from Brasquim (Porto Alegre-RS, Brazil).

\subsection{Nanostructured lipid carriers (NLC) preparation}

Phase inversion temperature (PIT) method was used as a low-energy approach to prepare nanostructured lipid carrier formulations [31]. Briefly, organic phase was composed of oil, solid lipid, and surfactants while aqueous phase consisted of purified water or phosphate buffer solution $\mathrm{pH} 7.4(10 \mathrm{mM})$. The phases were gradually heated at $80( \pm 5$ ${ }^{\circ} \mathrm{C}$ ) using a water bath and aqueous phase was added to organic phase under magnetic stirring and constant temperature $\left(80^{\circ} \mathrm{C}\right)$. After this temperature was reached, the lipid dispersion obtained was held for 5 min and then cooled down under continuous stirring. Both liquid lipid-to-solid lipid and surfactant-to-lipid weight ratios kept constant at 1.0 (Table 1) and sorbitan monooleate (SMO) and polyoxyethylene-40 hydrogenated castor oil (HCO-40) surfactants were mixed at constant ratio of 3.2:6.8 (wt \%), respectively. All formulations were prepared in triplicate $(n=18)$ and stored at $4\left( \pm 2{ }^{\circ} \mathrm{C}\right)$. Hesperitin-loaded NLC were prepared by adding different amounts of this flavanone in organic phase and using same procedure in order to prepare unloaded NLC. 
Table 1.

Composition (wt \%) of NLC formulations.

\begin{tabular}{ccclcl}
\hline NLCs & MCT & GB & Het & PW & PBS \\
\hline F1 & 5.0 & 5.0 & - & 80 & - \\
\hline F3 & 5.0 & 5.0 & - & - & 80 \\
F4 & 5.0 & 5.0 & 0.0625 & 80 & - \\
F5 & 5.0 & 5.0 & 0.0625 & - & 80 \\
F6 & 5.0 & 5.0 & 0.1875 & 80 & - \\
& 5.0 & 5.0 & 0.1875 & - & 80
\end{tabular}

MCT: medium chain triglycerides; GB: glyceryl behenate; Het: theoretical hesperitin concentration; PW: purified water; PBS: $10 \mathrm{mM}$ phosphate buffer solution $\mathrm{pH}$ 7.4.

\subsection{Particle size and polydispersity index analysis}

Hydrodynamic diameter of the NLC particles and polydispersity index (PdI) of the formulations were determined by photon correlation spectroscopy (PCS) at $25{ }^{\circ} \mathrm{C}$ and scattering angle of $173^{\circ}$ (Zetasizer ${ }^{\circledR}$ Nano ZS, Malvern PCS Instruments, UK). Samples were obtained by diluting $20 \mu \mathrm{L}$ with $2 \mathrm{~mL}$ ultrapure water. The reported values are average \pm SEM of three different batches of each colloidal dispersion.

\subsection{Zeta potential measurement}

Zeta $(\zeta)$ potential of NLC was measured by electrophoretic mobility using a Zetasizer ${ }^{\circledR}$ Nano ZS apparatus (Malvern PCS Instruments, UK). The Smoluchowski model was used to estimate $\zeta$ potential from electrophoretic mobility. The analyses were conducted at 25 
${ }^{\circ} \mathrm{C}$, and the samples were appropriately diluted (1/100) with ultrapurified water and $\mathrm{pH}$ measured. Values reported are average \pm SEM of three different batches of each colloidal dispersion.

\subsection{Stability study}

Stability study was performed by using the analytical centrifugation method with photometric detection at $865 \mathrm{~nm}$ (LUMiSizer ${ }^{\circledR}$ 611, LUM GmbH, Germany). The samples of unloaded NLC (F2), F4, and F6 formulations were incorporated into polycarbonate cell and equilibrated to $25^{\circ} \mathrm{C}$ before analyses. Measurements were carried out at 3,618 rpm for $4.5 \mathrm{~h}$ resulting in accelerated migration of the dispersed particles and integration of transmission profiles within a selected region of the holding cell allowed to get information about dispersion stability.

\subsection{Thermal analysis of NLC formulation}

Differential Scanning Calorimetry (DSC) analysis was performed using a DSC Q200 ${ }^{\circledR}$ Scanning Calorimeter (TA instruments ${ }^{\circledR}$ ). For this, $5.0 \mathrm{mg}$ of bulk lipid or freeze-dried samples of hesperitin-loaded NLC (F4 and F6) and unloaded NLC (F2) were weighed separately in a standard aluminum pan and hermetically sealed. An empty pan was used as the reference. The analyses were conducted under inert nitrogen atmospheric conditions at a flow rate of $50 \mathrm{~mL} \mathrm{~min}^{-1}$ and the samples were scanned at a constant rate of $5{ }^{\circ} \mathrm{C} \mathrm{min}-1$ from 20 to $120{ }^{\circ} \mathrm{C}$. The crystallinity degree (CD) was calculated as shown in Eq. (1) [29,30]:

$\mathrm{CD}(\%)=($ NLC melting enthalpy/melting enthalpy of the bulk lipid $\mathrm{x}$ lipid concentration in the NLC) $\mathrm{x} 100$ 


\subsection{Electron microscopy analyses}

NLC formulations were analyzed using transmission electron microscopy (TEM) technique. Aliquots $(\sim 100 \mu \mathrm{L})$ of hesperitin-loaded NLC (F4) were put onto copper TEM grids and excess sample was removed by filter paper. After that, the samples were dried at room condition before analyses. Transmission electron micrographs of the samples were taken using a Microscope HT7700 Hitachi (Fukuoka, Japan) operated at an accelerating voltage of $80 \mathrm{KV}$ and emission $12 \mu \mathrm{A}$.

\subsection{Hesperitin quantification in NLC, entrapment efficiency, and drug loading}

Hesperitin concentration was determined using a UV spectrophotometric method. In order to establish linearity of the proposed method three calibration curves were constructed at seven concentrations levels within the range of $3.0-15.0 \mu \mathrm{g} \mathrm{mL}^{-1}(\mathrm{n}=21)$. Least square regression analysis was done for the data (Optical density $=0.074$ [hesperitin, $\left.\left.\mu \mathrm{g} \mathrm{mL} L^{-1}\right]-0.027 ; \mathrm{r}=0.9987\right)$ and for all spectrophotometric analyses the Beer's law was obeyed. Hesperitin amount in NLC was assessed by dispersing a known amount of formulation in ethanol followed by centrifugation at 2,000 rpm for $10 \mathrm{~min}$. Afterwards, hesperitin concentration in the supernatant was determined by UV spectroscopy conducted on an Even UV/VIS spectrophotometer (Ionlab, Paraná, Brazil) with detection at $285 \mathrm{~nm}$. Free hesperitin content was determined by measuring the non-incorporated drug present in a clear ultrafiltrate obtained through separation of aqueous phase using an ultrafiltration/ultracentrifugation procedure (Microcon Ultracel YM-100, Millipore, Ireland) at 10,000 rpm for $1 \mathrm{~h}$, at $4{ }^{\circ} \mathrm{C}$ (Eppendorf, Centrifuge $5430 \mathrm{R}$, Hamburg, Germany). All analyses were performed in triplicate and the entrapment efficiency (E.E.) of hesperitin into NLC and drug loading (D.L.) were calculated from following equations: E.E. $(\%)=($ THet - FHet/ThHet $) \times 100$ 
Where, THet is the total hesperitin concentration; FHet, free hesperitin concentration; ThHet, theoretical hesperitin concentration.

D.L. $(\%)=($ Het encapsulated into NLC/amount of weighed NLC) $\times 100$

\subsection{In vitro release of hesperitin from NLC}

In vitro release experiments were carried out using the dialysis technique and under sink conditions. An aliquot $(4.0 \mathrm{~mL})$ of hesperitin-loaded NLC (F4) or free hesperitin (hydroethanolic dispersion $10 \% \mathrm{v} / \mathrm{v}$ ) was placed inside a dialysis bag (cellulose membrane, molecular weight cut-off 14,000, Sigma-Aldrich Co., St. Louis, MO), sealed, and immersed in a vessel containing $125 \mathrm{~mL}$ of $10 \mathrm{mM}$ phosphate buffer solution (PBS $\mathrm{pH}$ 7.4). The releasing system was maintained at $37 \pm 1^{\circ} \mathrm{C}$, under magnetic stirring (100 rpm). One-milliliter aliquots were taken out of the dissolution medium at pre-determined time intervals, replaced with fresh buffer solution (PBS), and analyzed for hesperitin release by spectrophotometry UV at $285 \mathrm{~nm}$. Release profile of hesperitin was expressed as cumulative amount of released hesperitin (average \pm SEM) and plotted versus time. The experiments were carried out in triplicate for both free and encapsulated hesperitin.

\subsection{Cell culture and cytotoxicity assay}

Human T98G glioblastoma cell line (ATCC CRL-1690) was cultivated in Dulbecco's Modified Eagle's Medium-Low (DMEM-LG; Gibco BRL, USA) supplemented with 2 mmol L ${ }^{-1}$ glutamine, $100 \mathrm{U} \mathrm{mL}^{-1}$ penicillin, $100 \mu \mathrm{g} \mathrm{mL} \mathrm{m}^{-1}$ streptomycin, $2.5 \mu \mathrm{g} \mathrm{mL}^{-1}$ amphotericin $\mathrm{B}$, and $10 \%$ fetal bovine serum (FBS) at $37{ }^{\circ} \mathrm{C}$ and $5 \% \mathrm{CO}_{2}$. In order to assess in vitro cytotoxic effect of free and nanoencapsulated hesperitin (F4 formulation) on glioblastoma cells, different concentrations of this flavanone were tested between 1.65 
and $36.39 \mu \mathrm{M}$. T98G cells were seeded in 96-well plate at density of $5 \times 10^{3}$ cells per well. After overnight adhesion, cells were treated with free hesperitin or hesperetin-loaded NLC for $24 \mathrm{~h}$, following the cell treatment every well was washed with PBS (pH 7.4) and proliferation MTT method was applied.

Cell MTT proliferation method [33] was employed for assessment of T98G cell viability after cytotoxicity assay. Briefly, aliquots of $20 \mu \mathrm{L}$ MTT solution $\left(5 \mathrm{mg} \mathrm{mL}^{-1}\right)$ and 180 $\mu \mathrm{L}$ medium without phenol red were added to each well. The cells were then incubated for $4 \mathrm{~h}$, at $37{ }^{\circ} \mathrm{C}$ and $5 \% \mathrm{CO}_{2}$ to allow the formazan-forming reaction. Following incubation, the medium containing MTT solution was removed, and formed formazan crystals were dissolved in 2-propanol. Optical density was measured at 570 and $690 \mathrm{~nm}$ using a Biotek Cytation 5 - Cell Imaging Multi-Mode Reader (Winooski, VT, USA). As positive controls T98G cells were incubated with unloaded NLC diluted at $2.0 \%(\mathrm{v} / \mathrm{v})$ or cell culture medium containing DMSO $0.5 \%(\mathrm{v} / \mathrm{v})$, while as a negative control, T98G cells were incubated only with cell culture medium. Results are presented as percentage of survival, taking the negative control (untreated cells) as $100 \%$.

\subsection{Statistical analysis}

All the experiments were performed in triplicate and data are expressed as average value $( \pm$ SEM). Statistical analysis was performed by One-way ANOVA followed by Tukey’s pairwise comparisons using the Prism GraphPad Software. The statistical significance was set at $\mathrm{p}<0.05$.

\section{Results and discussion}

Phenolic compounds present in different plants (fruits, vegetables, legumes, etc.) are the main bioactive molecules related to functional properties such as antioxidant, anti- 
inflammatory, antimicrobial, antiviral, and antineoplastic. However, biomedical application of these molecules has been adversely affected by their low aqueous solubility and poor stability in physiological environment resulting in a limited bioavailability. In order to overcome such limitations, several drug delivery systems have been developed to encapsulate flavonoids, coumarinea, stilbenes, tannins and among these systems are nanoemulsions, microemulsions, liposomes, polymeric nanoparticles, and lipid nanoparticles $[3,15,24,34]$. In this study, we prepared nanostructured lipid carriers containing hesperitin using a relatively simple and low-cost technique based low-energy emulsification approach, i.e. PIT method. Nanostructured lipid carrier formulations were characterized and the in vitro cytotoxic effect of NLC containing hesperitin was assessed on T98G glioblastoma cells.

\subsection{NLC characterization, stability study, electron microscopy, and thermal analysis} Average size, PdI, zeta potential, and $\mathrm{pH}$ values of NLC formulations tested are reported in Table 2. Both hesperitin-loaded NLC and unloaded NLC formulations varied in appearance from translucent to slightly turbid with bluish reflection typical of very fine colloidal dispersions [35]. This appearance could be attributed to poor light scattering by the relatively small lipid particles $(r<100 \mathrm{~nm})$ in formulations since all the NLC dispersions tested showed an average particle diameter below $80 \mathrm{~nm}$ (Table 2). In addition, both hesperitin entrapment into NLC and different dispersant phases used (PW or PBS) here had only a minor effect on particle size even at the highest concentration of this flavanone $(\mathrm{p}<0.05)$. Likewise, loaded and unloaded NLC formulations showed low PdI values $(<0.2)$ with a single peak in particle size distribution characteristic of homogeneous dispersions. In fact, F4 formulation showed a PdI value about 0.1 characterized by unimodal narrow size distribution (Fig. 1) confirming the highest quality 
of this colloidal dispersion. As reported by Bhattacharjee [36], colloidal dispersions with PdI values lower or equal to 0.1 are considered highly monodisperse while those with PdI values between 0.1 and 0.4 are moderately polydisperse. Therefore, the combination of SMO and HCO-40 nonionic surfactants resulted in achievement of very small homogeneous lipid particles $(<100 \mathrm{~nm})$ using a thermal method of emulsification. The formation of small lipid particles using PIT method is closely associated with changes in the nonionic surfactant structure as well as in its physicochemical characteristics, which occur during heating. At low temperatures, surfactant head-groups are highly hydrated and become gradually dehydrated under heating. All these temperature-dependent changes result in alterations of surfactant water solubility, along with its optimum curvature at interfacial monolayer. Above the PIT, the surfactant is mainly located in the organic phase and around the PIT there is similar affinity of surfactant between organic phase and aqueous phase, leading formation of bicontinuous microemulsion with very low interfacial curvature. The rapid cooling $(<\mathrm{PIT})$ of emulsified system results in microemulsion disruption and consequent surfactant movement from organic phase to aqueous phase forming very fine lipid particles $[21,23,37,38]$. 
Table 2.

Particle size, polydispersity index (PdI), zeta-potential, and pH of NLC formulations (average $\pm \operatorname{SEM}, \mathbf{n}=3$ ).

\begin{tabular}{ccccc}
\hline NLCs & Size $(\mathbf{n m})$ & PdI & $\begin{array}{c}\zeta \text { potential } \\
(\mathbf{m V})\end{array}$ & $\mathbf{p H}$ \\
& & & & \\
\hline F1 & $77.52( \pm 1.07)$ & $0.131( \pm 0.005)$ & $-19.70( \pm 1.14)$ & $7.94( \pm 0.08)$ \\
F2 & $69.75( \pm 2.19)$ & $0.122( \pm 0.030)$ & $-24.80( \pm 0.15)$ & $7.93( \pm 0.09)$ \\
F3 & $66.82( \pm 0.58)$ & $0.187( \pm 0.017)$ & $-17.80( \pm 1.69)$ & $6.25( \pm 0.05)$ \\
F4 & $66.17( \pm 1.34)$ & $0.099( \pm 0.004)$ & $-20.43( \pm 0.49)$ & $6.34( \pm 0.11)$ \\
F5 & $76.74( \pm 0.99)$ & $0.140( \pm 0.003)$ & $-17.20( \pm 0.44)$ & $7.51( \pm 0.04)$ \\
& & & & \\
\hline & $63.90( \pm 0.49)$ & $0.079( \pm 0.009)$ & $-19.07( \pm 0.92)$ & $6.98( \pm 0.09)$ \\
\hline
\end{tabular}




\section{Figure 1.}

Average diameter of particles and PdI from F2 (A), F4 (B), and F6 (C) formulations.

Normalized NIR transmission profiles obtained with F2 (D), F4 (E), and F6 (F)

formulations by forced stability analysis during analytical centrifugation. The first and the last registered profiles are shown in red and in green, respectively.
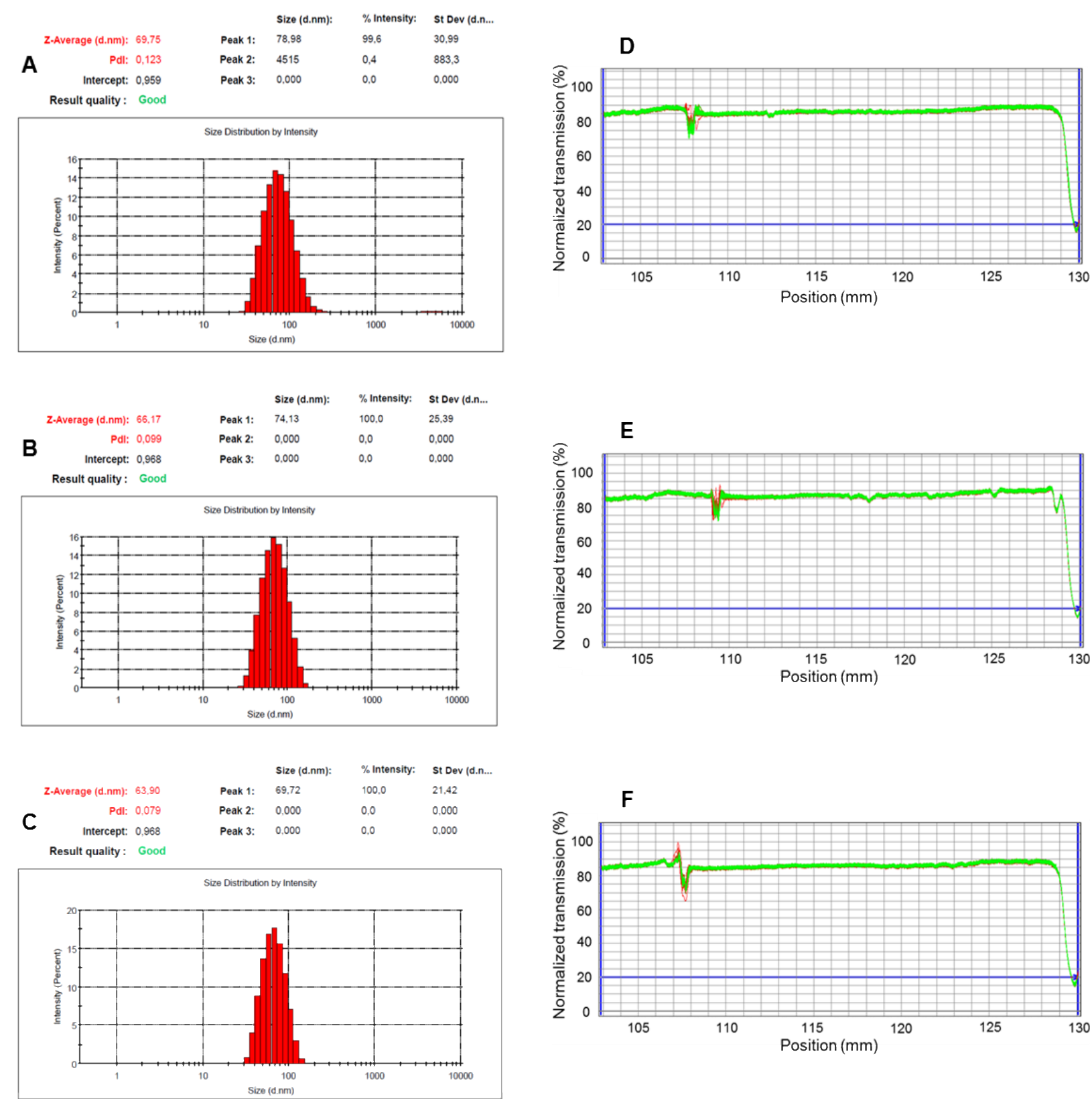
Electrokinetic potential or $\zeta$ potential of colloid particles was measured by electrophoretic mobility technique. For all formulations, $\zeta$ potential values were significantly negative $(\sim$ $-20 \mathrm{mV}$ ) and there was no statistically significant difference between electric potential of particles from different NLC formulations $(\mathrm{p}>0.05)$. These $\zeta$ potential values indicate high physical stability of lipid dispersions due to electrostatic repulsive forces among colloid particles preventing rapid agglomeration and sedimentation or flotation [36].

Stability studies were carried out for unloaded NLC (F2), F4, and F6 formulations using analytical photocentrifugation method. This method is based on measurement of particle migration and aggregation from colloid dispersions by application of a centrifugal force and photometric detection. The kinetics of the particle separation process is characterized by shape and progression of light transmission profiles measured as a function of time and position across the entire sample length. The first and the last registered profiles are shown in red and in green, respectively. A total of 255 transmission profiles were recorded in intervals of $65 \mathrm{~s}$. As can be observed in Fig. 1, there were no significant differences in light transmission profiles among lipid dispersions (F2, F4, and F6) during all analysis time and throughout sample. Moreover, high transmittances (about $85 \%$ ) for NLC samples were maintained during analysis, i.e. there were no instability signals such as particle agglomeration, sedimentation/flotation of dispersed phase, or visible phase separation. Moreover, F4 formulation presented distribution (D) of particle migration velocity at D10 \%, D50 \%, and D90 \% of 9.169, 9.391, and $10.163 \mu \mathrm{m} \mathrm{s}^{-1}$, respectively, and mean velocity $9.591 \mu \mathrm{m} \mathrm{s}^{-1}( \pm 0.392)$. Such narrow velocity distribution, i.e. only slight speed variation of particle migration between D10 \% and D90 \%, indicates good physical stability of hesperitin-loaded NLC. According to these results, it could be to claim that encapsulation of this flavanone into NLC was not able to alter physical stability of lipid formulations even at the highest flavanone concentration. Therefore, results 
obtained from NLC stability study could be mainly explained due to gravitational stability of nanoparticles, i.e. smaller particles $(<90 \mathrm{~nm})$ in these dispersions may be under influence of Brownian motion only [24,35]. In general, NLC produced herein could remain stable for 12 months when stored at $25^{\circ} \mathrm{C}$.

DSC analyses were performed for bulk glyceryl behenate powder and freeze-dried formulation samples (F2, F4, and F6). Fig. 2 shows the DSC heating thermographs ranging from 20 to $120{ }^{\circ} \mathrm{C}$ and Table 3 summarizes the data extracted from calorimetric thermograms. During heating, for all samples, main peaks were centered between 67.0 and $72.0{ }^{\circ} \mathrm{C}$. Bulk glyceryl behenate exhibited an endothermic peak at $71.9^{\circ} \mathrm{C}$, this melting temperature is characteristic of the most stable form of glyceryl behenate, i.e. $\beta$ ' polymorphic form $[31,32]$. On the other hand, for lipid nanocarriers (F2, F4, and F6) were observed less intense endothermic peaks with lower melting temperatures than the bulk lipid. Therefore, the main components of NLC formulation (lipids, surfactants, and hesperitin) altered crystallinity degree of solid lipid matrix as compared to pure glyceryl behenate (Table 3). It has been widely reported that the addition of surfactant(s) and liquid lipid(s) to NLC formulation as well as when transforming bulk lipid in nanoparticle might result in melting point depression and broadening peaks. These events could occur due to interactions between solid lipid(s) and liquid lipid(s) and/or surfactants [16,31,32]. Moreover, the reduction of onset, enthalpy, melting temperature, and crystallinity degree from F2, F4, and F6 formulations suggests also that hesperitin was mostly entrapped into lipid core of nanocarriers affecting crystallization process of solid lipid. It is worth mentioning that this change in lipid matrix from NLC might enhance drug entrapment efficiency decreasing expulsion of drug during storage when compared to SLN [16-18]. In view of results from physicochemical characterization, stability study, and thermal analysis described above, F4 NLC formulation was chosen to further assays due to its 
better stability profile as well as this formulation was prepared with $\mathrm{PBS}(\mathrm{pH} 7.4)$ as aqueous phase, which is an isotonic solution and non-toxic to most cells.

\section{Figure 2.}

DSC thermograms of GB bulk lipid, F2, F4, and F6 NLC formulations.

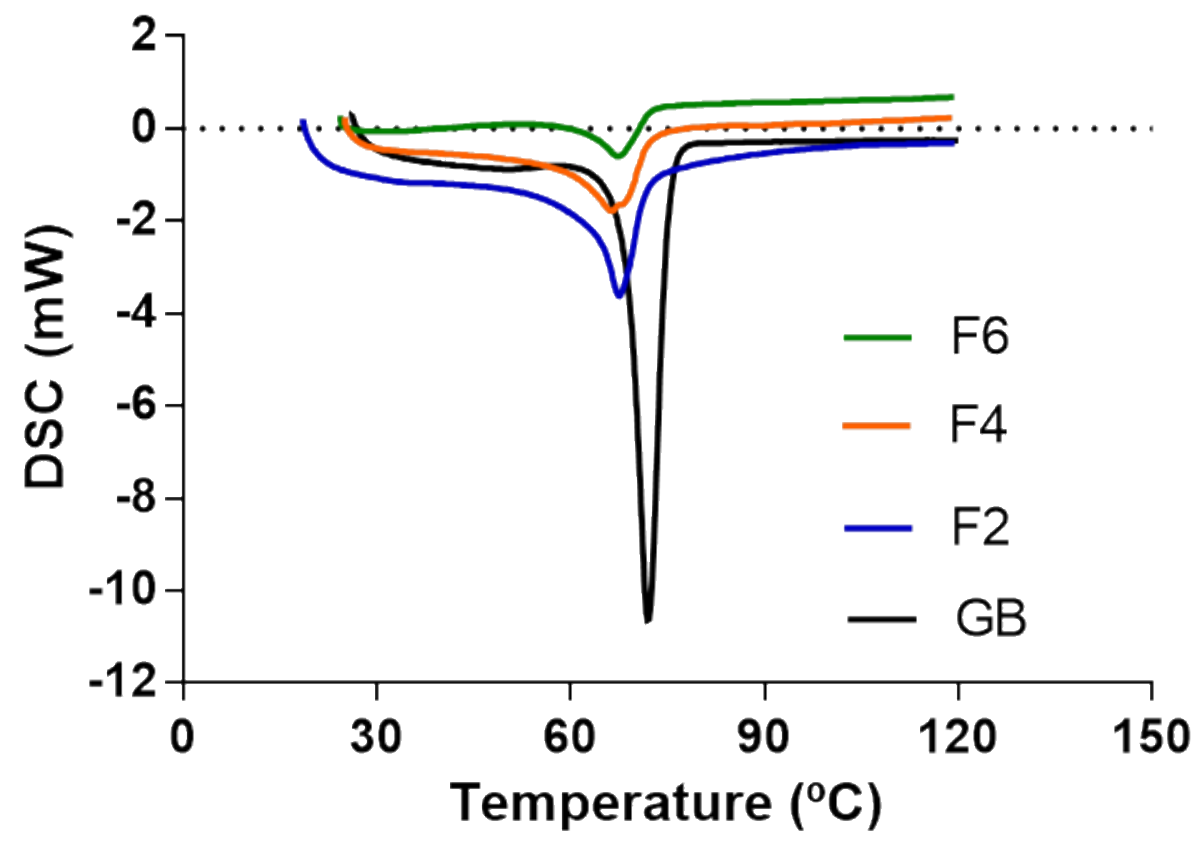


Table 3.

Parameters extracted from the DSC thermograms of the bulk glyceryl behenate and NLC dispersions.

\begin{tabular}{ccccc}
\hline Samples & $\begin{array}{c}\text { Melting } \\
\text { temperature } \\
\left({ }^{\mathbf{0}} \mathbf{C}\right)\end{array}$ & Onset $\left({ }^{\mathbf{0}} \mathbf{C}\right)$ & $\begin{array}{c}\text { Enthalpy } \\
\left(\mathbf{J ~ g}^{-\mathbf{1}}\right)\end{array}$ & $\begin{array}{c}\text { Crystallinity } \\
\text { degree }(\%)\end{array}$ \\
\hline bulk GB & 71.9 & 68.8 & -136.3 & 100.0 \\
F2 & 67.8 & 64.0 & -83.1 & 61.1 \\
F4 & 67.7 & 65.3 & -80.7 & 59.2 \\
F6 & 66.9 & 63.0 & -22.1 & 16.2 \\
\hline
\end{tabular}

Transmission electron microscopy (TEM) has been widely applied to characterize particle matrix structure and diameter of nanostructures [18]. TEM analyses were carried out for F4 NLC formulation and micrographs are shown in Fig. 3. According to photomicrographs, there was no particle aggregation and lipid nanoparticles had almost spherical shapes with a particle size of less $100 \mathrm{~nm}$ and homogeneous size distribution. These findings are in accord with earlier results from particle size analyses using photon correlation spectroscopy. Moreover, the matrix from lipid core was highly heterogeneous presenting lipid cluster domains compatible with multiple oil-in-solid fat-in-water type of NLC $[17,18]$. It is worth noting that the drying process and imperfect crystallization of lipids might result in sample deformation. 


\section{Figure 3.}

TEM photomicrographs from hesperitin-loaded NLC F4 formulation. Magnifications (A) x7.0K, (B) x12.0K, and (C) x20.0K.
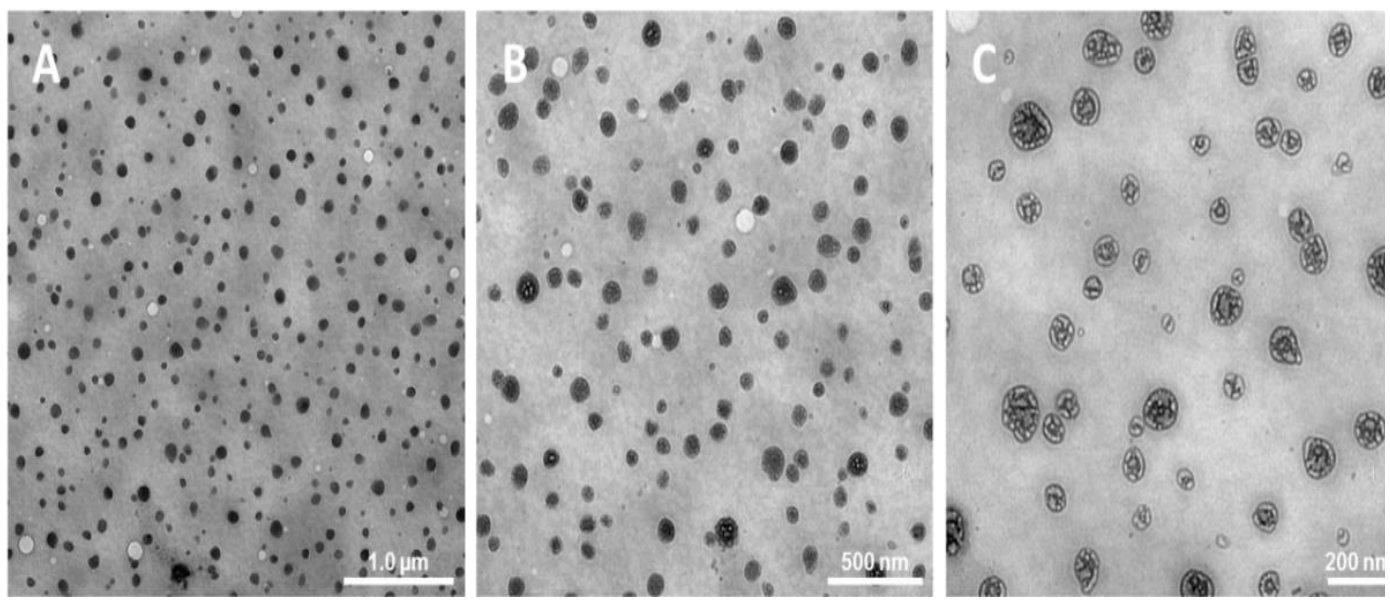

\subsection{Hesperitin quantification, entrapment efficiency, and in vitro release study}

Quantitative analyses of hesperitin into NLC formulation were carried out using UV spectrophotometric method. F4 NLC formulation was assessed for total amount, entrapment efficiency, drug loading, and in vitro release of hesperitin. Total concentration of this flavanone found for NLC formulation was $0.55 \mathrm{mg} \mathrm{mL}^{-1}( \pm 0.01)$. Meanwhile, entrapment efficiency of hesperitin into NLC and drug loading reached $72.7 \%( \pm 0.92)$ and $3.48 \%( \pm 0.17)$, respectively, confirming a better capacity of this kind lipid dispersion for encapsulation of hydrophobic drugs.

Dialysis bag diffusion technique was chosen to assess and compare hesperitin release from hydroethanolic dispersion (free flavanone) and NLC formulation. Release profiles of free and encapsulated hesperitin in PBS pH 7.4 are shown in Fig. 4. Comparing both release profiles, free hesperitin was released 2.5-fold faster than flavanone entrapped into lipid nanocarriers, and thus it was observed a sustained release profile of hesperitin from NLC formulation. Almost all content of free hesperitin (90\%) was released in the first 
$24 \mathrm{~h}$ of essay while from NLC after $72 \mathrm{~h}$ only $63 \%( \pm 2.0)$ of hesperitin were released. Moreover, it was not noticed a burst release of hesperitin from NLC at initial phase demonstrating that there was no drug adsorbed to particle surface. These results propose that hesperitin might be entrapped into lipid core of NLC, which results in slow drug release from formulation. The release profile of hesperitin from NLC in PBS pH 7.4 was kinetically evaluated by zero-order $\left(r^{2}=0.9616\right)$, first-order $\left(r^{2}=0.7613\right)$, Higuchi $\left(r^{2}=\right.$ 0.9964), and Rigter-Peppas $\left(r^{2}=0.8488\right)$ models. The best-fit model was Higuchi that describes the hesperitin release from NLC formulation as a process controlled by Fickian diffusion mechanism. 


\section{Figure 4.}

In vitro release profiles of hesperitin from NLC formulation (F4) and hydroethanolic dispersion (free hesperitin) in PBS pH 7.4 (average \pm SD, $\mathbf{n}=3$ ). Release profiles: (A) shows during $72 \mathrm{~h}$ and (B) in the first $12 \mathrm{~h}$.

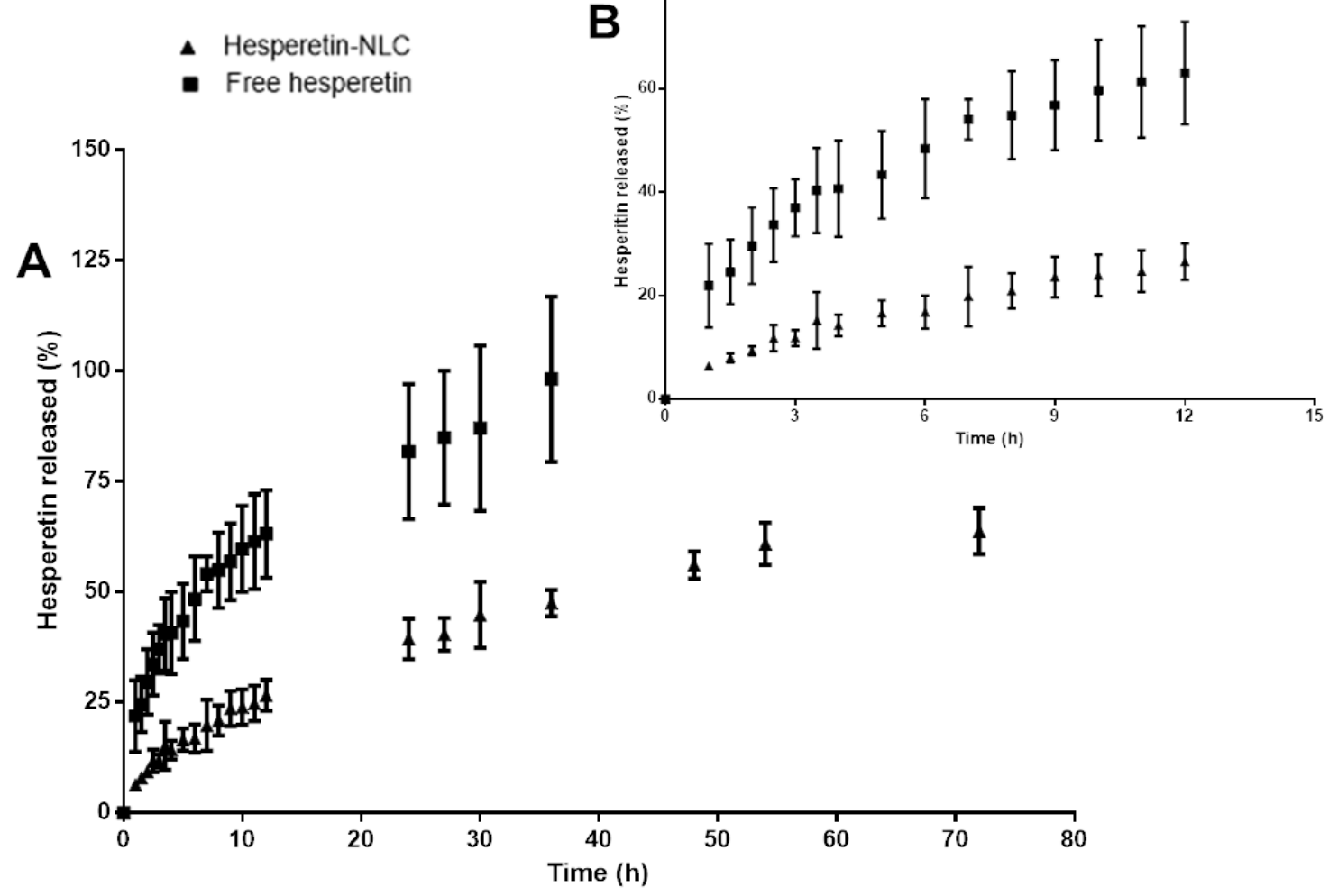

\subsection{In vitro cytotoxicity assessment}

The in vitro cytotoxic effect of free hesperitin and hesperitin-loaded NLC was assessed on T98G glioblastoma cells following $24 \mathrm{~h}$ exposure to flavanone concentrations ranging from 1.65 to $36.39 \mu \mathrm{M}$. The results are presented in Fig. 5 .

For both free and encapsulated hesperitin, T98G cell viability decreased in a dose dependent manner. After $24 \mathrm{~h}$ of treatment of glioblastoma cells, there was no evidence of cytotoxic effect for free hesperitin and hesperitin-loaded NLC at flavanone concentrations below $33.08 \mu \mathrm{M}$ and $26.47 \mu \mathrm{M}$, respectively, as compared with negative 
control cells (NC). However, at higher concentrations (33.08 and $36.39 \mu \mathrm{M})$ of encapsulated hesperitin, the T98G cell viability was significantly affected with a reduction in viable cell percentage levels for less than $10 \%$ in comparison with untreated cells. In addition, hesperitin-loaded NLC demonstrated also higher cytotoxic activity as compared to free hesperitin at $29.77,33.08$, and $36.39 \mu \mathrm{M}$. Therefore, the NLC formulation containing hesperitin led to a great decrease in cell viability at the highest flavanone concentrations tested. Yoo et al. [39] assessed in vitro cytotoxicty of hesperidin isolated from Euodia daniellii on XF-498 cell line (human CNS solid tumor) and $\mathrm{IC}_{50}$ found was higher than $30 \mu \mathrm{M}$ following $48 \mathrm{~h}$ exposure to this glycoside. Recently, Ersoz et al. [12] assessed anticancer activity of hesperitin-loaded polymeric nanoparticles against C6 glioma cells and $\mathrm{IC}_{50}$ of hesperitin loaded nanoparticles was 2.0-fold lower than free hesperitin as well as dose- and time-dependent. Thus all these findings revel that encapsulation of hesperitin into nanoparticles could enhance cytotoxic effect on neoplastic cells. This improved antitumor efficacy of hesperitin combination in polymeric or lipid nanoparticles might be mainly related to better internalization of these nanocarriers by cells [12]. 


\section{Figure 5.}

Viability of T98G glioblastoma cells, using MTT assay, after 24 h-exposure to free hesperitin or hepseritin-loaded NLC. Data are shown as average \pm SEM $(n=3)$ and statistical analysis by one-way ANOVA with Tukey posthoc test $p<0.05$ (NC= negative control; $\mathrm{PC}=$ positive controls; $(@)$ p $<0.01$ with $\mathrm{PC}$ and free Hesperitin concentrations of $16.54,19.85$ and $26.47 \mu \mathrm{M}$; $(*) p<0.05$ with NLC-Hesperitin at $26.47 \mu \mathrm{M} ;(\$) \mathrm{p}<0.001$ with $\mathrm{NC}$, free Hesperitin concentrations between $1.65-13.23$ $\mu M$, at 29.77 $\mu \mathrm{M}$ and 33.08 $\mu \mathrm{M}$ and with NLC-Hesperitin concentrations between 1.65 - $19.85 \mu \mathrm{M}$; (\#) $\mathrm{p}<0.001$ with $\mathrm{PC}, \mathrm{NC}$, all free Hesperitin concentrations and NLC-Hesperitin concentrations between 1.65 - 26.47 $\mu \mathrm{M})$.

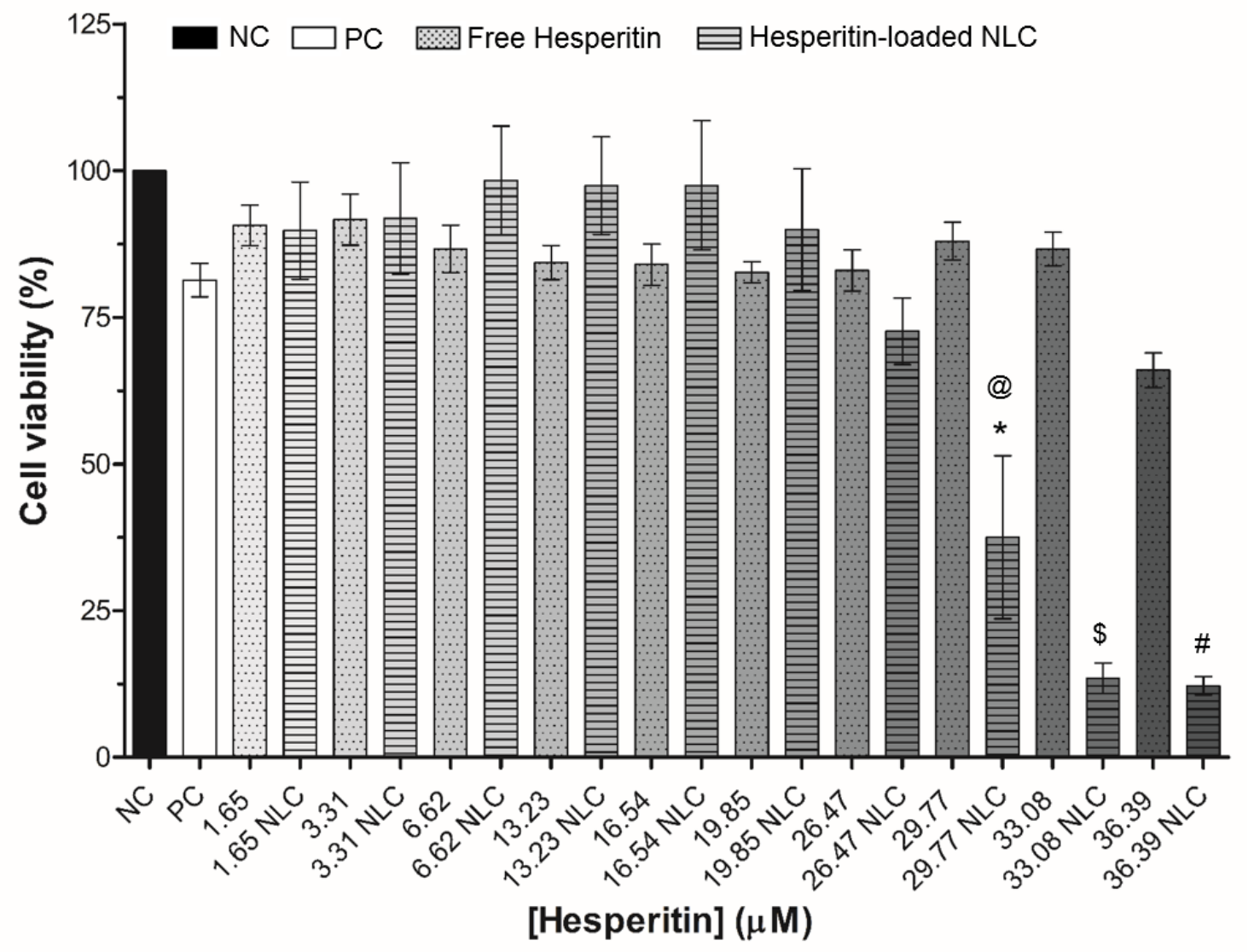




\section{Conclusions}

In summary, we have successfully encapsulated hesperitin into NLC formulation using PIT method, which is a low-energy emulsification approach. TEM analyses were in accord with PCS results showing that the nanoparticles obtained had almost spherical shapes with a heterogeneous lipid matrix structure and diameter below $100 \mathrm{~nm}$. Accelerated stability study confirmed the good physical stability of F4 NLC formulation with minimal variation in lipid particle migration rate. In addition, $\mathrm{F} 4$ formulation reached an encapsulation efficiency of hesperitin about $70 \%$ as well as the in vitro release assay demonstrated that this flavanone could be released in a sustained manner from NLC. Cytotoxicity study revealed that hesperitin-loaded NLC was able significantly to reduce cell viability of $\mathrm{T} 98 \mathrm{G}$ cells at $33 \mu \mathrm{M}$ of flavanone when compared to free hesperitin. Therefore, these results might confirm NLC formulation as an effective flavanone delivery system for application in different treatments. Moreover, such findings encourage further studies on NLC containing flavonoids for use in the therapeutics protocols against other neoplastic cell lines.

\section{Acknowledgements}

This study was supported by CAPES/COFECUB agreement (\#Ph-C 859-15). M.P.S.M. and T.D.H. thank Science and Technology Support Foundation of Pernambuco State (FACEPE \#BIC-3173-4.03/16). A.C.T. thanks the Brazilian Federal Agency for Support and Evaluation of Graduate Education (CAPES), the State of São Paulo Research Foundation (FAPESP) Thematic project \#2013/50181-1 \& Project FINEP 01.10.0758.01, and the National Council for Scientific and Technological Development (CNPq) for their fi013/501 support Project PRONON-SIPAR \#25000.077093/2015-86. H.L.P thanks FAPESP project \#2017/16356-0 and Project PRONON-SIPAR \#25000.077093/2015-86. 
We also thank the National Institute of Science and Technology (INCT) of Nanobiotechnology project \#573880/2008-5 for their financial support.

\section{References}

[1] A. Miranda, M. Blanco-Prieto, J. Sousa, A. Pais, C. Vitorino, Breaching barriers in glioblastoma. Part II: Targeted drug delivery and lipid nanoparticles, Int. J. Pharm. 531 (2017) 389-410, https://doi.org/10.1016/j.ijpharm.2017.07.049.

[2] C.P. Haar, P. Hebbar, G.C. Wallace, A. Das, W.A. Vandergrift, J.A. Smith, P. Giglio, S.J. Patel, S.K. Ray, N.L. Banik, Drug Resistance in Glioblastoma: A Mini Review, Neurochem. Res. 37 (2012) 1192-1200, https://doi.org/10.1007/s11064-011-0701-1.

[3] A. Faridi Esfanjani, E. Assadpour, S.M. Jafari, Improving the bioavailability of phenolic compounds by loading them within lipid-based nanocarriers, Trends Food Sci. Technol. 76 (2018) 56-66, https://doi.org/10.1016/j.tifs.2018.04.002.

[4] M.K. Khan, Zill-E-Huma, O. Dangles, A comprehensive review on flavanones, the major citrus polyphenols, J. Food Compos. Anal. 33 (2014) 85-104, https://doi.org/10.1016/j.jfca.2013.11.004.

[5] B.B. Chandrika, M. Steephan, T.R. Santhosh Kumar, A. Sabu, M. Haridas, Hesperetin and Naringenin sensitize HER2 positive cancer cells to death by serving as HER2 Tyrosine Kinase inhibitors, Life Sci. $160 \quad$ (2016) 47-56, https://doi.org/10.1016/j.lfs.2016.07.007.

[6] R. Jayaraman, S. Subramani, H.S.H. Abdullah, M. Udaiyar, Antihyperglycemic effect of hesperetin, a citrus flavonoid, extenuates hyperglycemia and exploring the potential role in antioxidant and antihyperlipidemic in streptozotocin-induced diabetic rats, Biomed. Pharmacother. 97 (2018) 98-106, https://doi.org/10.1016/j.biopha.2017.10.102. 
[7] E. Kheradmand, A. Hajizadeh Moghaddam, M. Zare, Neuroprotective effect of hesperetin and nano-hesperetin on recognition memory impairment and the elevated oxygen stress in rat model of Alzheimer's disease, Biomed. Pharmacother. 97 (2018) 1096-1101, https://doi.org/10.1016/j.biopha.2017.11.047.

[8] A. Sassi, I.M. Bzéouich, N. Mustapha, M. Maatouk, K. Ghedira, L. Chekir-Ghedira, Immunomodulatory potential of hesperetin and chrysin through the cellular and humoral response, Eur. J. Pharmacol. $812 \quad$ (2017) 91-96, https://doi.org/10.1016/j.ejphar.2017.07.017.

[9] J.P. Ferreira de Oliveira, C. Santos, E. Fernandes, Therapeutic potential of hesperidin and its aglycone hesperetin: Cell cycle regulation and apoptosis induction in cancer models, Phytomedicine (2019) in press. https://doi.org/10.1016/j.phymed.2019.152887.

[10] G. Krishnan, J. Subramaniyan, P.C. Subramani, B. Muralidharan, D. Thiruvengadam, Hesperetin conjugated PEGylated gold nanoparticles exploring the potential role in anti-inflammation and anti-proliferation during diethylnitrosamineinduced hepatocarcinogenesis in rats, Asian J. Pharm. Sci. 12 (2017) 442-455, https://doi.org/10.1016/j.ajps.2017.04.001.

[11] M. Tsirigotis-Maniecka, Ł. Lamch, I. Chojnacka, R. Gancarz, K.A. Wilk, Microencapsulation of hesperidin in polyelectrolyte complex microbeads: Physicochemical evaluation and release behavior, J. Food Eng. 214 (2017) 104-116, https://doi.org/10.1016/j.jfoodeng.2017.06.015.

[12] M. Ersoz, A. Erdemir, D. Duranoglu, D. Uzunoglu, T. Arasoglu, S. Derman, B. Mansuroglu, Comparative evaluation of hesperetin loaded nanoparticles for anticancer activity against C6 glioma cancer cells, Artif. Cells Nanomed. Biotechnol. 47 (2019) 319_ 329, https://doi.org/10.1080/21691401.2018.1556213. 
[13] A.R. Bilia, B. Isacchi, C. Righeschi, C. Guccione, M.C. Bergonzi, Flavonoids Loaded in Nanocarriers: An Opportunity to Increase Oral Bioavailability and Bioefficacy, Food Nutr. Sci. 5 (2014) 1212-1227, https://doi.org/10.4236/fns.2014.513132.

[14] D. Duranoğlu, D. Uzunoglu, B. Mansuroglu, T. Arasoglu, S. Derman, Synthesis of hesperetin-loaded PLGA nanoparticles by two different experimental design methods and biological evaluation of optimized nanoparticles, Nanotechnol. 29 (2018) 395603, https://doi.org/10.1088/1361-6528/aad111.

[15] M. Estanqueiro, M.H. Amaral, J. Conceição, J.M.S. Lobo, Nanotechnological carriers for cancer chemotherapy: The state of the art, Colloids Surf. B Biointerfaces 126 (2015) 631-648, https://doi.org/10.1016/j.colsurfb.2014.12.041.

[16] A. Gordillo-Galeano, C.E. Mora-Huertas, Solid lipid nanoparticles and nanostructured lipid carriers: A review emphasizing on particle structure and drug release, Eur. J. Pharm. Biopharm. $133 \quad$ (2018) 285-308, https://doi.org/10.1016/j.ejpb.2018.10.017.

[17] A. Khosa, S. Reddi, R.N. Saha, Nanostructured lipid carriers for site-specific drug delivery, Biomed. Pharmacother. $103 \quad$ (2018) 598-613, https://doi.org/10.1016/j.biopha.2018.04.055.

[18] V.R. Salvi, P. Pawar, Nanostructured lipid carriers (NLC) system: A novel drug targeting carrier, J. Drug Deliv. Sci. Technol. 51 (2019) 255-267, https://doi.org/10.1016/j.jddst.2019.02.017.

[19] A. Beloqui, M.A. Solinís, A. Rodríguez-Gascón, A.J. Almeida, V. Véronique Préat, Nanostructured lipid carriers: Promising drug delivery systems for future clinics, Nanomedicine 12 (2016) 143-161, https://doi.org/10.1016/j.nano.2015.09.004. 
[20] N. Anton, J.-P. Benoit, P. Saulnier, Design and production of nanoparticles formulated from nano-emulsion templates-A review, J. Control. Release 128 (2008) 185-199, https://doi.org/10.1016/j.jconrel.2008.02.007.

[21] N. Anton, T.F. Vandamme, The universality of low-energy nano-emulsification, Int. J. Pharm. 377 (2009) 142-147, https://doi.org/10.1016/j.ijpharm.2009.05.014.

[22] J.S. Komaiko, D.J. McClements, Formation of Food-Grade Nanoemulsions Using Low-Energy Preparation Methods: A Review of Available Methods, Compr. Rev. Food Sci. Food Saf. 15 (2016) 331-352, https://doi.org/10.1111/1541-4337.12189.

[23] S. Gao, D.J. McClements, Formation and stability of solid lipid nanoparticles fabricated using phase inversion temperature method, Colloids Surf. A Physicochem. Eng. Asp. 499 (2016) 79-87, https://doi.org/10.1016/j.colsurfa.2016.03.065.

[24] D.J. McClements, Delivery by Design (DbD): A Standardized Approach to the Development of Efficacious Nanoparticle- and Microparticle-Based Delivery Systems, Compr. Rev. Food Sci. Food Saf. 17 (2018) 200-219, https://doi.org/10.1111/15414337.12313.

[25] T. Tadros, P. Izquierdo, J. Esquena, C. Solans, Formation and stability of nanoemulsions, Adv. Colloid Interface Sci. 108-109 (2004) 303-318, https://doi.org/10.1016/j.cis.2003.10.023.

[26] C. Solans, I. Solé, Nano-emulsions: Formation by low-energy methods, Curr. Opin. Colloid Interface Sci. 17 (2012) 246-254, https://doi.org/10.1016/j.cocis.2012.07.003.

[27] D.J. McClements, Edible nanoemulsions: fabrication, properties, and functional performance, Soft Matter. 7 (2011) 2297-2316, https://doi.org/10.1039/c0sm00549e.

[28] M. Fathi, J. Varshosaz, M. Mohebbi, F. Shahidi, Hesperetin-Loaded Solid Lipid Nanoparticles and Nanostructure Lipid Carriers for Food Fortification: Preparation, 
Characterization, and Modeling, Food Bioprocess Technol. 6 (2013) 1464-1475, https://doi.org/10.1007/s11947-012-0845-2.

[29] C. Ott, I. Lacatusu, G. Badea, I.A. Grafu, D. Istrati, N. Babeanu, R. Stan, N. Badea, A. Meghea, Exploitation of amaranth oil fractions enriched in squalene for dual delivery of hydrophilic and lipophilic actives, Ind. Crops Prod. 77 (2015) 342-352, http://dx.doi.org/10.1016/j.indcrop.2015.08.057.

[30] S. Saad, I. Ahmad, S.M. Kawish, U.A. Khan, F.J. Ahmad, A. Ali, G.K. Jain, Improved cardioprotective effects of hesperidin solid lipid nanoparticles prepared by supercritical antisolvent technology, Colloids Surf. B Biointerfaces 187 (2020) 110628, https://doi.org/10.1016/j.colsurfb.2019.110628.

[31] P.L. Goto, M.P. Siqueira-Moura, A.C. Tedesco, Application of aluminum chloride phthalocyanine-loaded solid lipid nanoparticles for photodynamic inactivation of melanoma cells, Int. J. Pharm. 518 (2017) 228-241, https://doi.org/10.1016/j.ijpharm.2017.01.004.

[32] S. Carvalho, C.M. Noronha, C.L. Floriani, R.C. Lino, G. Rocha, I.C. Bellettini, P.J. Ogliari, P.L.M. Barreto, Optimization of $\alpha$-tocopherol loaded solid lipid nanoparticles by central composite design, Ind. Crop. Prod. 49 (2013) 278-285, http://dx.doi.org/10.1016/j.indcrop.2013.04.054.

[33] F. Denizot, R. J. Lang, Modifications to the tetrazolium dye procedure giving improved sensitivity and reliability, J. Immunol. Methods 89 (1986) 271-277, https://doi.org/10.1016/0022-1759(86)90368-6.

[34] B.V. Bonifácio, P.B. Silva, M.A.S. Ramos, K.M.S. Negri, T.M. Bauab, M. Chorilli, Nanotechnology-based drug delivery systems and herbal medicines: a review, Int. J. Nanomed. 9 (2014) 1-15, https://doi.org/10.2147/IJN.S52634. 
[35] D.J. McClements, Nanoemulsions versus microemulsions: terminology, differences, and similarities, Soft Matter. 8 (2012) 1719-1729, https://doi.org/10.1039/C2SM06903B. [36] S. Bhattacharjee, DLS and zeta potential - What they are and what they are not?, J. Control. Release 235 (2016) 337-351, https://doi.org/10.1016/j.jconrel.2016.06.017. [37] C. Carbone, C. Martins-Gomes, C. Caddeo, A.M. Silva, T. Musumeci, R. Pignatello, G. Puglisi, E.B. Souto, Mediterranean essential oils as precious matrix components and active ingredients of lipid nanoparticles, Int. J. Pharm. 548 (2018) 217-226, https://doi.org/10.1016/j.ijpharm.2018.06.064.

[38] P. Chuesiang, U. Siripatrawan, R. Sanguandeekul, L. McLandsborough, D.J. McClements, Optimization of cinnamon oil nanoemulsions using phase inversion temperature method: Impact of oil phase composition and surfactant concentration, J. Colloid Interface Sci. 514 (2018) 208-216, https://doi.org/10.1016/j.jcis.2017.11.084. [39] S.W. Yoo, J.S. Kim, S.S. Kang, K.H. Son, H.W. Chang, H.P. Kim, K. Bae, C.-O. Lee, Constituents of the Fruits and Leaves of Euodia daniellii, Arch. Pharm. Res. 25 (2002) 824-830, https://doi.org/10.1007/BF02976999. 